\title{
A Rare Presentation of a Large Embryonal Rhabdomyosarcoma at Retroperitoneal Region with Intra-thoracic Extension \& Misdiagnosed as Lower Respiratory Tract Infection: A Case Report
}

\author{
Sachin S Kadam ${ }^{1, *}$, Pradeep Tripathi' ${ }^{2}$ Tejaswini Kadam ${ }^{3}$ \\ ${ }^{1}$ Department of Surgical Oncology, Vedant Cancer \& Multispeciality Hospital, Mumbai, India \\ ${ }^{2}$ Department of Bariatric \& Laparoscopic Surgery, Vedant Cancer \& Multispeciality Hospital, Mumbai, India \\ ${ }^{3}$ Department of Ophthalmology, Conwest \& Jain Superspeciality Eye Hospital, Mumbai, India
}

Received December 7, 2020; Revised February 15, 2021; Accepted February 24, 2021

\section{Cite This Paper in the following Citation Styles}

(a): [1] Sachin S Kadam, Pradeep Tripathi, Tejaswini Kadam, " A Rare Presentation of a Large Embryonal Rhabdomyosarcoma at Retroperitoneal Region with Intra-thoracic Extension \& Misdiagnosed as Lower Respiratory Tract Infection: A Case Report," Cancer and Oncology Research, Vol. 7, No. 1, pp. 1 - 9, 2021. DOI: 10.13189/cor.2021.070101.

(b): Sachin S Kadam, Pradeep Tripathi, Tejaswini Kadam (2021). A Rare Presentation of a Large Embryonal Rhabdomyosarcoma at Retroperitoneal Region with Intra-thoracic Extension \& Misdiagnosed as Lower Respiratory Tract Infection: A Case Report. Cancer and Oncology Research, 7(1), 1 - 9. DOI: 10.13189/cor.2021.070101.

Copyright $\odot 2021$ by authors, all rights reserved. Authors agree that this article remains permanently open access under the terms of the Creative Commons Attribution License 4.0 International License

\begin{abstract}
Introduction: We are reporting a case of 18 year old boy who presented with features of lower respiratory tract infections and labelled as suffering from left suprarenal gland tumor. However, after complete evaluation, it is diagnosed as left retroperitoneal tumor extending into left thoracic cavity with involvement of left lower lobe lung. He underwent debulking surgery. Rhabdomyosarcoma (RMS) originates from immature striated muscle and it is considered as the most aggressive malignant mesenchymal tumor. The most common location of RMS is head and neck region. The retroperitoneal presentation of embryonal RMS is extremely rare. The four histological features of RMS, classified by WHO are embryonal, alveolar, pleomorphic and spindle cell or sclerosing. Pleomorphic RMS has worse prognosis. The incidence of embryonal tumors is higher in males with bimodal age distribution, between 2-6 years and second peak between 10-18 years. Their detection is incidental when the size is small and as the size enlarges, symptoms helps in detection. Due to its rarity at retroperitoneal location, there is a lack of literature over the adjuvant treatment. As the size enlarges at retroperitoneal region, enblock resection with clear margins becomes a
\end{abstract}

difficult task and if planned for R0 resection, multiorgan resection escalates surgical morbidity rate. Conclusion: It is a rare location of embryonal rhabdomyosarcoma at retroperitoneal region with rare presentation of intra-thoracic infiltration. R0 resection is the principle goal of surgical excision of retroperitoneal sarcoma.

Keywords Embryonal Rhabdomyosarcoma, Retroperitoneal, Intra-Thoracic, LRTI, R0 Resection, Case Report

\section{Introduction}

Retroperitoneal Sarcomas (RPS ) constitutes only 10 $-15 \%$ of all soft tissue sarcomas [1]. Surveillance, Epidemiology, and End Results (SEER) database had studied a population based series and they found that the average annual incidence of RPS was approximately 2.7 cases per million population [2]. Rhabdomyosarcoma (RMS ) originates from immature striated muscle and it is considered as the most aggressive malignant mesenchymal 
tumor [ 3 ]. Out of all adult malignancies, soft tissue sarcomas accounts for more than $1 \%$ of malignancies and RMS accounts for 3\% of all soft tissue sarcomas [ 4 ]. RMS is split up into four subtypes depending upon its histological features which is classified by WHO and the subtypes are embryonal, alveolar, pleomorphic and spindle cell or sclerosing [ 5 ]. The incidence of embryonal tumors is higher in males and that of alveolar tumors is slightly higher in blacks [ 6 ]. Its presentation also differs in adults and young population [ 7 ]. There is bimodal age distribution of embryonal RMS, between 2-6 years and second peak between 10-18 years and uncommon after 45 years [ 8 ]. The pleomorphic subtype carries worse prognosis and it is most commonly found in adults [ 9 ]. The most common location of RMS is head and neck region ( $38 \%$ ) followed by genitourinary tract ( $22 \%$ ) and extremities $(18 \%)$ and other less common site includes the trunk, chest wall, perineal, anal region, the retroperitoneum and biliary tract. The 5-year survival rate of RMS has increased from $53 \%$ to $67 \%$ for children younger than 15 years and from $30 \%$ to $51 \%$ for adolescents aged 15 to 19 years [ 10 ]. The retroperitoneal presentation of embryonal RMS is extremely rare. Most of the times these tumors are asymptomatic. Clinical presentation depends upon the size of the tumor, its local infiltrative features and surrounding compression effects. We are reporting a case of 18 year old boy who presented with features of lower respiratory tract infections and labelled as suffering from left suprarenal gland tumor. However, after complete evaluation, diagnosed as left retroperitoneal tumor extending into left thoracic cavity with involvement of left lower lobe lung.

\section{Case Report}

18 year old boy with Eastern Co-operative Oncology Group Performance Status I ( ECOG PS -I ) with no comorbidity presented with history of on and off fever, productive cough for the last 3 months. There was no supportive relevant medical, surgical, familial or congenital history. Patient had consultation with a local clinician and he had been diagnosed as suffering from lower respiratory tract infections. He had been treated according to his symptomology with higher antibiotics, antipyretics and other supportive measures. After 2 months of following conservative treatment he didn't get relief from the symptoms. A chest $\mathrm{X}$ ray was advised by the treating clinician and radio-opacity at left lung lower lobe was picked up. With these findings, patient was referred to chest physician for further management. The treating physician changed the antibiotic course and called him after two weeks. Still the situation was status quo with no relief from the symptoms. Just for curiosity, the physician had advised him to do ultrasound of abdomen. On ultrasound, the new finding of left enlarged suprarenal gland was detected and the boy was referred to our clinic for management of left adrenal gland tumor.

When the patient visited to our clinic, he had fever spike. Therefore, we admitted him and initially planned to optimize him. We optimized the patient with intravenous antipyretics, antibiotics and fluid support. On clinical examination of respiratory system, air entry was markedly absent at left lower zone and on palpation of abdomen a large,vague, hard, fix mass was felt at left side of abdomen with involvement of epigastrium, left hypochondriac, part of umbilical and left lumbar quadrants. Rest of the general examination was unremarkable. The ultrasound report was suggestive of a large left enlarged adrenal gland of size $25 \times 20 \times 15 \mathrm{~cm}$ with involvement of left hemidiaphragm and left renal hilum. However, classical symptoms of adrenal pheochromocytoma or paraganglioma ( i.e. episodic hypertension, unexplained sweating, flushing ) were absent. Our next question was to rule out the functional status of the tumor as to plan further investigation (CECT or MRI). It was important as there were chances of anaphylaxis due to contrast injection if CECT was preferred. In that case, MRI abdomen with pelvis was the safer option. Hence we investigated him with 24 hour urinary metanephrine and catecholamine estimation. Urinary levels were within normal range which ruled out functional status. We advised him to undergo repeat chest $\mathrm{x}$ ray and Contrast Enhanced Computed Tomography ( CECT ) of thorax, abdomen and pelvis. Chest Xray had radioopacity at left lower zone with decreased expansion of left lung as compared to right lung [ Fig. 1 ]. CECT report [ Fig. $2 \& 3$ ] was indicative of a large heterogeneously enlarged lesion of size measuring $26 \times 20 \times 14 \mathrm{~cm}$ arising from left adrenal gland as the gland was not seen separately from the mass. Cranially it was infiltrating the diaphragm with possibility of left lower lobe lung involvement and medially it was extended till the renal hilum with abutment of renal vessels with no retroperitoneal lymphadenopathy and no distant metastasis. It's an uncommon presentation of adrenal tumor of this size with local infiltrative features in a young boy. Therefore, we were in dilemma and not convinced with radiology interpretation. We reviewed the serial sections of the scan multiple times and discussed with senior and experienced radiologist. Ultimately, we detected a plane between the lesion and upper pole of kidney and left adrenal gland which was indicative of non-origin of the mas from left kidney or left adrenal. The possible differential diagnosis were sarcoma, extraadrenal paraganglioma or lymph node mass. In a young boy with this presentation, it was a rare possibility of paraganglioma. Lymph node mass with this type of local infiltrative features is a rare finding in a young boy. Retroperitoneal sarcoma was the most probable diagnosis considering his age and presentation. 


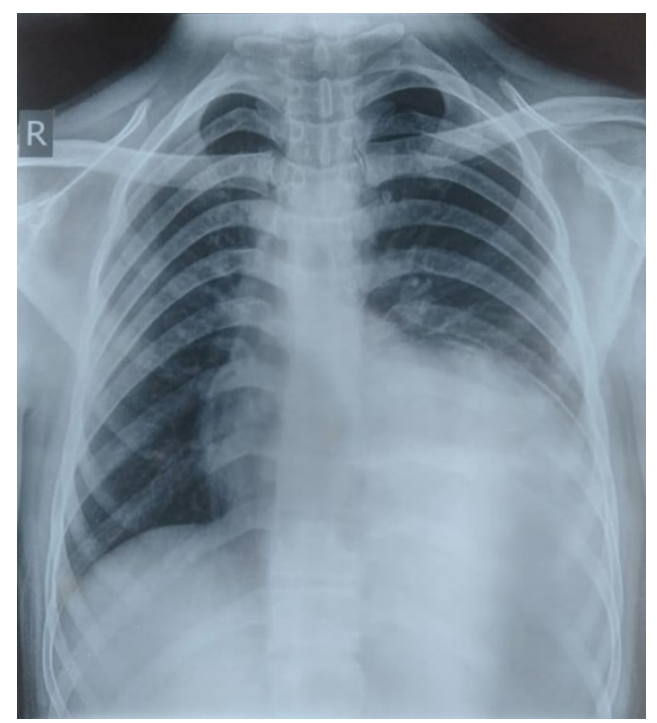

Figure 1. Xray chest - infiltration of left lung lower lobe by RPS

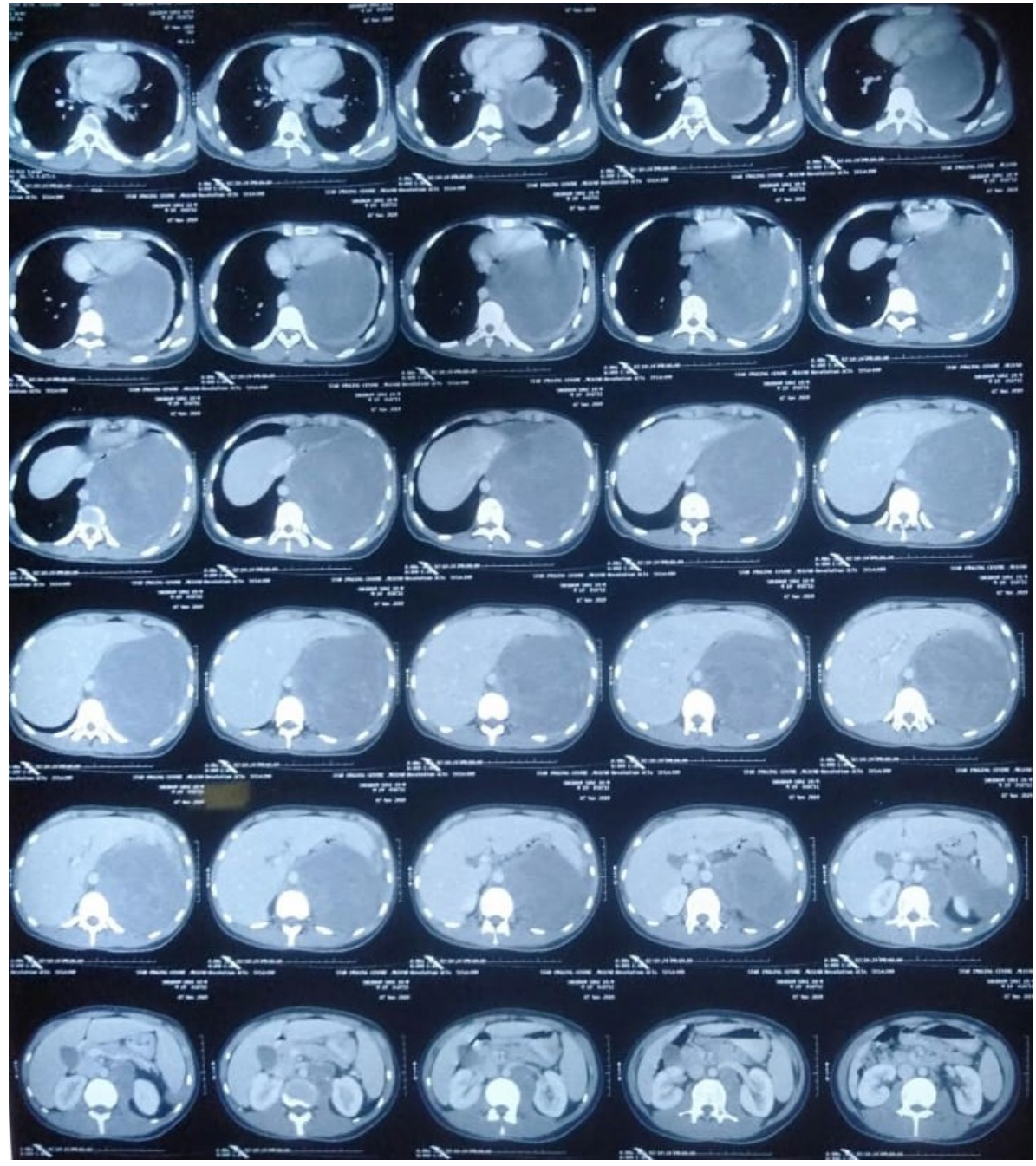

Figure 2. Retroperitoneal RMS - Axial view 


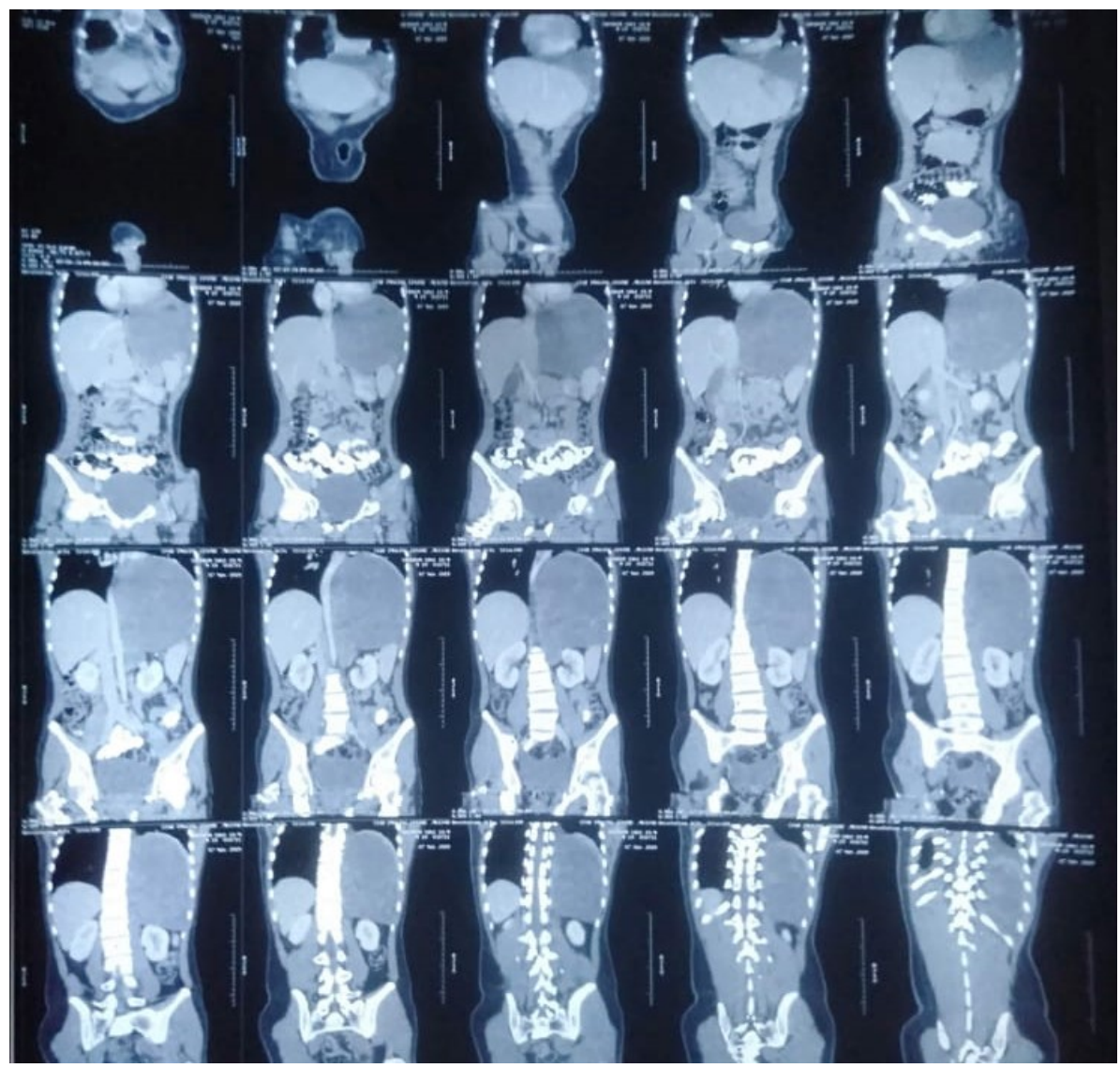

Figure 3. Retroperitoneal RMS - coronal view

Case was discussed in our institutional multidisciplinary tumor board. It was an operable disease with possibility of multiorgan resection. The definitive diagnosis was possible only after histopathological examination. The point of discussion was whether there is any neoadjuvant treatment for this case to downstage the disease. Adrenal or renal tumors lacks the literature support of neoadjuvant treatment. Retroperitoneal sarcoma has supporting evidences of neoadjuvant chemotherapy and neoadjuvant radiotherapy. However to start neoadjuvant treatment without biopsy was unethical and biopsy, itself was the invitation for seeding of tumor along needle tract. Hence, our board decided to go ahead with radical surgery with plan of multiorgan resection to achieve R0 resection.

Patient was optimized in the ward with control of fever, nutritional support and lung expanding respiratory exercises. Consent of multiorgan resection and post operative ventilatory support had been taken. After six days of optimization, he was posted for surgery. With midline laparotomy incision, abdomen was explored. Intraoperative, the hard and fixed mass was present as described in the scan with medial extension at left renal hilum and cranial extension to diaphragm. The mass was well encapsulated, hard and fixed posteriorly with vague extensions. Hence, we decided to do the debulking of the tumor as enblockn removal was difficult. Medially, there was only abutment to the renal vessels with no infiltration, so we removed it completely with preservation of left kidney and adrenal gland. Cranially, we resected the diaphragm with adequate macroscopic margins. The part of left lung lower lobe was infiltrated by the tumor, hence we resected a segment of lung parenchyma along with tumor with the use of linear stapler. Diaphragm was reconstructed with bio absorbable mesh and clips were applied at the site of tumor for radiotherapy planning. Two intercostal drainage tubes were inserted, one anteriorly to avoid pneumothorax and other posteriorly for drainage of fluids. Posterior tube was removed after 48 hours. Patient was observed in intensive and critical care unit for 3 days and shifted to ward on $4^{\text {th }}$ postoperative day (POD ). Oral feeding was started from 2 nd postoperative day. Anterior intercostal drainage tube was removed on $7^{\text {th }}$ postoperative day and patient was discharged on $9^{\text {th }}$ POD after confirming lung expansion on left side. Histopathology report was suggestive of embryonal rhabdomyosarcoma with moderate differentiation with infiltration of left lung lower lobe and diaphragm. Grossly, the tumor had glistening, gelatinous, fleshy tissues with areas of cyst formation, haemorrhage and necrosis. Tumor was positive for MyoD1, Myogenin A and Desmin. On Microscopic examination [ Fig. 4, 5 ], there were presence of densely packed hypercellular areas on the left side with less cellular myxoid area on the right and there was a mixture of small, undifferentiated, hyperchromatic round cells with 
differentiated eosinophilic cells (rhabdomyoblasts) with scanty collagen and myxoid stroma. In view of debulking with microscopic positive margin, chances of local recurrence were high. Our tumor board members had different opinions on adjuvant treatment for this case. Finally with available literature support, adjuvant chemotherapy with three drug regimen ( VAC ) to counter the metastatic potential of the tumor followed by adjuvant radiation therapy to avoid local recurrence was planned. Patient and his relatives were explained about the adjuvant treatment plan. However, patient didn't receive the treatment and lost follow up. We tried to trace all available contact information of the patient, but he was not traceable. After 9 months of surgery, patient revisited our clinic and presented with back pain.We re-evaluated him with PET $\mathrm{CT}$ and there was no local or distant recurrence. He was managed symptomatically as there was no role of adjuvant treatment after a long gap of 9 months. Patient is on periodic follow up with us according to our institutional follow up protocol and after one year of completion of the treatment, he is disease free.

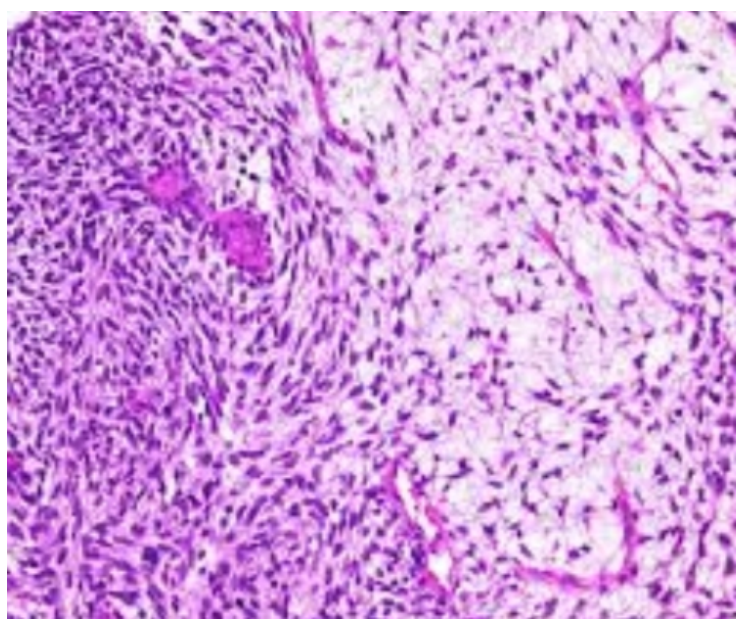

Figure 4. Microscopic appearance of Embryonal RMS (Low Magnification )

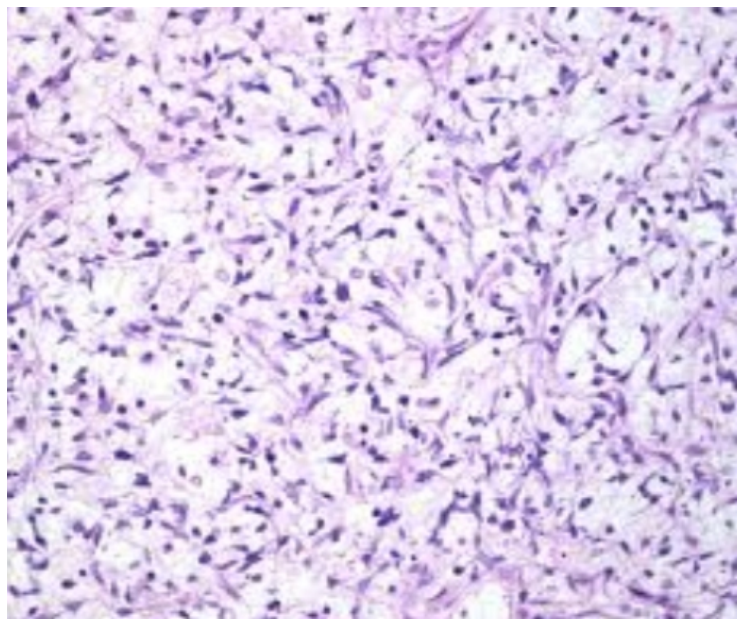

Figure 5. Microscopic appearance of Embryonal RMS ( High Magnification )

\section{Discussion}

Radiographic Imagings are the principle component for evaluation of retroperitoneal masses. Contrast-enhanced computed tomography (CECT) is the preferred radiographic diagnostic investigation which helps in defining the primary tumor with distant metastatic disease. MRI with gadolinium is reserved for patients with an allergy to iodinated contrast agents or if there is equivocal muscle, bone, or foraminal involvement. A role for positron emission tomography (PET) with fluorodeoxyglucose (FDG) in the initial staging evaluation is not yet established. The standard criterion which defines unresectable retroperitoneal sarcoma on the basis of radiographic imaging includes [ 11 ].

- Peritoneal deposits

- Extensive vascular involvement (aorta, vena cava, and/or iliac vessels). Involvement of the vena cava and iliac veins is a relative contraindication as these vessels can often be ligated or replaced with interposition grafts.

- Involvement of the root of the mesentery (specifically, the superior mesenteric vessels).

- Distant metastases that are not potentially resectable for cure.

- Spinal cord involvement

When the diagnosis is in doubt or if preoperative therapy is planned, the biopsy is clearly indicated. However several data supports percutaneous core needle biopsy of RPS and reported that tumor seeding of the biopsy tract is very rare [ 12,13 ].Therefore its up to the treating clinician's team or institutional protocol that decides the choice of biopsy. However, initial surgery without biopsy is an acceptable alternative if the radiographic diagnosis of RPS seems certain based on available imaging. We did the same with this young boy after confirming the diagnosis on radiographic imaging.

Previously, separate staging system for RPS was not available and common staging of Soft tissue sarcoma was used for it. The most recent AJCC (eighth edition, 2017) version has separate $\mathrm{T}$ stage classifications and prognostic stage groupings for RPS [Table 1, 14 ]. Current Children's Oncology Group Soft Tissue Sarcoma ( COG-STS ) protocols for rhabdomyosarcoma uses the TNM-based pretreatment staging system [ Table 2,15 ]. There is a lack of high level evidence for the treatment, evaluation and management of RPS due to its rare presentation. Neither the National Comprehensive Cancer Network (NCCN) nor the European Society of Medical Oncology (ESMO) provides guidance in selecting treatment for individual patients with RPS. The role of radiation therapy and chemotherapy, either given preoperatively or postoperatively, continues to be debated, and there is no consensus as to the best approach for all patients. The primary oncologic goal during surgical removal of RPS is microscopically negative (R0) resection. However, the 
large size of most RPS, with the inability to obtain wide margins due to anatomical barriers makes this goal difficult and the tumor is grossly removed but the margins are microscopically positive [16 ]. In the index case also, we removed the tumor grossly but there were chances of microscopic positive margins.

Table 1. Retroperitoneal soft tissue sarcoma TNM staging AJCC UICC 8th edition

\begin{tabular}{|c|c|}
\hline \multicolumn{2}{|c|}{ Primary tumor $(T), T$ category, $T$ criteria } \\
\hline $\mathrm{TX}$ & Primary tumor cannot be assessed \\
\hline T0 & No evidence of primary tumor \\
\hline $\mathrm{T} 1$ & Tumor $5 \mathrm{~cm}$ or less in greatest \\
\hline $\mathrm{T} 2$ & Tumor more than $5 \mathrm{~cm}$ and less than or equal to $10 \mathrm{~cm}$ in greatest dimension \\
\hline $\mathrm{T} 3$ & Tumor more than $10 \mathrm{~cm}$ and less than or equal to $15 \mathrm{~cm}$ in greatest dimension \\
\hline $\mathrm{T} 4$ & Tumor more than $15 \mathrm{~cm}$ in greatest dimension \\
\hline \multicolumn{2}{|c|}{ Regional lymph nodes $(\mathrm{N}), \mathrm{N}$ category, $\mathrm{N}$ criteria } \\
\hline No & No regional lymph node metastasis or unknown lymph node status \\
\hline N1 & Regional lymph node metastasis \\
\hline \multicolumn{2}{|c|}{ Distant metastasis $(M), M$ category, $M$ criteria } \\
\hline M0 & No distant metastasis \\
\hline M1 & Distant metastasis \\
\hline \multicolumn{2}{|c|}{ Definition of grade (G), $G$ definition } \\
\hline GX & Grade cannot be assessed \\
\hline G1 & Total differentiation, mitotic count and necrosis score of 2 or 3 \\
\hline G2 & Total differentiation, mitotic count and necrosis score of 4 or 5 \\
\hline G3 & Total differentiation, mitotic count and necrosis score of 6,7, or 8 \\
\hline \multicolumn{2}{|c|}{ Prognostic stage groups } \\
\hline IA & T1 N0 M0 G1, GX \\
\hline IB & T2, T3, T4 N0 M0 G1, GX \\
\hline II & T1 N0 M0 G2, G3 \\
\hline IIIA & T2 N0 M0 G2, G3 \\
\hline IIB & $\begin{array}{l}\text { T3, T4 N0 M0 G2, G3 } \\
\text { Any T N1 M0 Any G }\end{array}$ \\
\hline IV & Any T Any N M1 Any G \\
\hline
\end{tabular}

Table 2. COG-STS TNM criteria

\begin{tabular}{|c|c|}
\hline Favourable site & Orbit; nonparameningeal head and neck; genitourinary tract other than kidney, bladder, and prostate; biliary tract. \\
\hline Unfavourable site & Any site other than a favourable site. \\
\hline T1 & Tumor confined to organ or tissue of origin (noninvasive). \\
\hline T2 & Tumor extension beyond the organ or tissue of origin (invasive). \\
\hline a & Tumor $\leq 5 \mathrm{~cm}$ in maximum dimension. \\
\hline b & Tumor $>5 \mathrm{~cm}$ in maximum dimension. \\
\hline No & No clinical regional lymph node involvement. \\
\hline N1 & Clinical regional lymph node involvement. \\
\hline NX & Regional lymph nodes not examined; no information. \\
\hline M0 & No metastatic disease \\
\hline M1 & Metastatic disease \\
\hline
\end{tabular}

$\mathrm{T}=$ primary tumor $\mathrm{N}=$ regional lymph node $; \mathrm{M}=$ distant metastasis . 
Intergroup Rhabdomyosarcoma Studies-I (IRS-I), IRS-II, IRS-III, IRS-IV prescribed treatment plans on the basis of the Surgical-pathologic Group system and groups are defined by the extent of disease,by the completeness or extent of initial surgical resection after pathologic review of the tumor specimen [Table 3, 17-19 ]. Patients are classified for protocol purposes depending upon stage and Surgical-pathologic Group into a low risk, intermediate risk and high risk of disease recurrence [ Table 4, 20, 21 ]. The index case falls into intermediate risk group as histology was embryonal with large size, absence of regional lymph nodes and non-metastatic disease. Approximately $50 \%$ of patients with retroperitoneal RMS falls in the intermediate-risk category. The treatment plan of childhood retroperitoneal RMS is extrapolated from COG-STS protocols and we followed the same in the present case as lack of uniform consensus on childhood retroperitoneal RMS. Some of the case series had proved that initial chemotherapy followed by surgery with or without adjuvant radiation therapy improves overall survival in childhood intra-abdominal /retroperitoneal RMS [ 22, 23 ]. In the absence of preoperative biopsy with diagnostic dilemma, we did surgical excision of the lesion for the index case. VAC (Vincristine, Dactinomycin, and Cyclophosphamide ) is the standard multiagent chemotherapy regimen used for this category and there is no difference in outcome if two drugs with ifosfamide is used an alkylating agent [ 19 ]. The COG-D9803 clinical trial used topotecan as an additional course to VAC therapy for newly diagnosed patients with intermediate-risk disease. However the results with topotecan were not fare as compared with VAC alone [ 24 ]. The role of adjuvant chemotherapy in retroperitoneal RMS, post surgical resection is still not clear. Our tumor board members had different opinions on adjuvant chemotherapy. However, due to non availability of uniform consensus and limited literature, board decided to plan adjuvant chemotherapy for the index case as there were chances of distant recurrences in near future.

The preoperative radiation therapy ( RT ) has definitive advantage in local control of the RPS. It is very well tolerated and there is lower incidence of late irreversible side effects [ 25 ]. The main advantage is that gross tumor volume (GTV) can be precisely defined for RT planning which directs accurate targeting of RT around the tumor. The tumor itself displaces small bowel from the high-dose radiation treatment volume which in turn helps in accurate targeting with safer and less toxic treatment [ 26 ]. The role of intraoperative RT in local control of RPS had been proved by some of the non-randomized trials [ 27, 28 ]. The role of adjuvant RT in RPS is still an unclear chapter. Post surgical resection, which patient should receive adjuvant RT is still a debated question. Whether patients with microscopically positive margin are at increased risk of local recurrence or it really has an impact on survival is studied by some retrospective case series and all the available evidences had conflicting results. Series from M D Anderson and other case series lacks the evidence of impact on survival if there is microscopically positive margin [ 29-32 ]. Therefore, Adjuvant RT is an option for RPS with R0/R1 resection with high- or intermediate-grade tumors that are at risk for local recurrence. Hence, index case had been planned with adjuvant RT in view of high risk for local recurrence. However, in current clinical practice, most of the institutes follows surveillance without any adjuvant treatment as it is difficult to deliver postoperative adjuvant RT with acceptable morbidity and if any chance, the tumor recurs during surveillance, re-resection is advisable.

Table 3. Soft Tissue Sarcoma Committee of the Children's Oncology Group: Surgical-pathologic Group System

\begin{tabular}{|c|c|c|}
\hline Group & Incidence & Defination \\
\hline I & $\begin{array}{c}\text { Approximately } \\
13 \%\end{array}$ & $\begin{array}{l}\text { Localized tumor, completely removed } \\
\text { with microscopically clear margins } \\
\text { and no regional lymph node } \\
\text { involvement. }\end{array}$ \\
\hline II & $\begin{array}{c}\text { Approximately } \\
20 \%\end{array}$ & $\begin{array}{l}\text { Localized tumor, completely removed } \\
\text { with: (a) microscopic residual disease; } \\
\text { (b) regional disease with involved, } \\
\text { grossly removed regional lymph } \\
\text { nodes; or (c) regional disease with } \\
\text { involved nodes, grossly removed but } \\
\text { with microscopic residual and/or } \\
\text { histologic involvement of the most } \\
\text { distal node from the primary tumor. }\end{array}$ \\
\hline III & $\begin{array}{c}\text { Approximately } \\
48 \%\end{array}$ & $\begin{array}{l}\text { Localized tumor, incompletely } \\
\text { removed with gross, residual disease } \\
\text { after: (a) biopsy only or (b) subtotal } \\
\text { resection. }\end{array}$ \\
\hline IV & $\begin{array}{c}\text { Approximately } \\
18 \%\end{array}$ & $\begin{array}{l}\text { Distant metastases present at } \\
\text { diagnosis. This category includes: (a) } \\
\text { radiographically } \\
\text { identified evidence of tumor spread or } \\
\text { (b) positive tumor cells in cerebral } \\
\text { spinal fluid, pleural or peritoneal } \\
\text { fluids, or implants in these regions. }\end{array}$ \\
\hline
\end{tabular}

Table 4. Soft Tissue Sarcoma Committee of the Children's Oncology Group: Rhabdomyosarcoma Risk Group Classification Based on the Ongoing ARST1431 Trial

\begin{tabular}{|c|c|c|}
\hline Histology & Stage & Group \\
\hline Low Risk & 1 & I, II, III (orbit only) \\
\hline Embryonal & 2 & I, II \\
\hline Embryonal 2 I, II & 1 & III ( nonorbit ) \\
\hline Intermediate Risk & \multicolumn{2}{|c|}{ III } \\
\hline Embryonal & 3 & I, II \\
\hline Embryonal & 4 & IV (age $<10$ years ) \\
\hline Embryonal & $1,2,3$ & I, II, III \\
\hline Embryonal & & IV \\
\hline Alveolar & 4 & IV (age $>/=10$ years ) \\
\hline High Risk & 4 &
\end{tabular}




\section{Conclusions}

It is a rare presentation of an embryonal rhabdomyosarcoma at retroperitoneal region with intrathoracic extension. The principle goal of surgical excision of retroperitoneal sarcoma is to achieve complete R0 resection with acceptable multiorgan resection morbidity. In the absence of uniform consensus over the treatment of retroperitoneal rhabdomyosarcoma, the institutional multidisciplinary sarcoma / tumor board has a definitive role in planning the treatment with best possible oncological outcomes. Locally advanced RPS has a risk of local or distant recurrence. Hence patients with RPS requires standard follow up protocol or institutional follow up protocol.

\section{REFERENCES}

[1] Raut CP, Pisters PW, " Retroperitoneal sarcomas: Combined-modality treatment approaches,' J Surg Oncol, 2006; 94:81.

[2] Porter GA, Baxter NN, Pisters PW, " Retroperitoneal sarcoma: a population-based analysis of epidemiology,surgery, and radiotherapy,' Cancer, 2006, 106:1610.

[3] Furlong MA, Mentzel T, Fanburg-smith JC, ' Pleomorphic rhabdomyosarcoma in adults : A clinicopathological study of 38 with emphasis on morphological variants and recent skeletal muscle specific markers," Mod Patholo., 2001 ; 14 : 595-603

[4] Weiss SW, Goldblum JR, Flope AL. Enzinger,Weiss, '” soft tissue tumors - 5 th edition," Philadelphia : Mosby, 2007. p.601-38.

[5] Fletcher CDM, Bridge JA, Hogendoorn PCW et al, ' WHO Classification of Tumors of soft Tissue and Bone," 4th Edition, Volume 5, France : IARC Press ; 2013, p. 127-34.

[6] Smith MA, Altekruse SF, Adamson PC et al, " Declining childhood and adolescent cancer mortality," Cancer, 2014, 120(16): 2497-506

[7] Sultan I, Qaddoumi I, Yaser S et al, ' Comparing adult and pediatric rhabdomyosarcoma in the surveillance, epidemiology and end results programme,'” 1973 to 2005 , an analysis of 2,600 patients, J Clin Oncol. 2009; 273391

[8] Goldblum, J. R., Weiss, S. W., \& Flope, A. L. (2019), Enzinger, Weiss, "' Soft Tissue Tumors - Seventh Edition," Philadelphia, PA. Elsevier

[9] Alkhormi AM, Alqifari A, Aljarbou OZ et al, " Primary duodenal embryonal rhabdomyosarcoma in adults : a case report,"' AME Case Rep, 2019, $3: 29$.

[10] Ognjanovic S, Linabery AM, Charbonneau B et al, ' ' Trends in childhood rhabdomyosarcoma incidence and survival in the United States,' 1975-2005, Cancer 115 (18 ), 2009, 4218-26.
[11] Jaques DP, Coit DG, Hajdu SI, Brennan MF, ' Management of primary and recurrent soft-tissue sarcoma of the retroperitoneum," Ann Surg, 1990, 212:51.

[12] Wilkinson MJ, Martin JL, Khan AA et al, " Percutaneous core needle biopsy in retroperitoneal sarcomas does not influence local recurrence or overall survival, " Ann Surg Oncol, 2015, 22:853.

[13] Berger-Richardson D, Swallow CJ, " Needle tract seeding after percutaneous biopsy of sarcoma: Risk/benefit considerations, ' Cancer 2017,123:560.

[14] Pollock RE, Maki RG, Baldini EH et al, " Soft Tissue Sarcoma of the Retroperitoneum, In: AJCC Cancer Staging Manual," 8th, Amin, MB (Eds), AJCC, Chicago 2017. p.531.

[15] Lawrence W, Anderson JR, Gehan EA et al, ' Pretreatment TNM staging of childhood rhabdomyosarcoma: a report of the Intergroup Rhabdomyosarcoma Study Group, Children's Cancer Study Group, Pediatric Oncology Group,' Cancer 80 (6), 1997, 1165-70.

[16] Anaya DA, Lev DC, Pollock RE, "' The role of surgical margin status in retroperitoneal sarcoma," J Surg Oncol 2008, 98:607.

[17] Crist WM, Garnsey L, Beltangady MS et al, ' Prognosis in children with rhabdomyosarcoma: a report of the intergroup rhabdomyosarcoma studies I and II, Intergroup Rhabdomyosarcoma Committee," J Clin Oncol, 1990, 8 (3):443-52.

[18] Crist W, Gehan EA, Ragab AH et al, ' The Third Intergroup Rhabdomyosarcoma Study," J Clin Oncol, 1995,13 (3):610-30.

[19] Crist WM, Anderson JR, Meza JL et al, " Intergroup rhabdomyosarcoma study-IV: results for patients with nonmetastatic disease," J Clin Oncol, 2001,19 (12): 3091-102.

[20] Raney RB, Anderson JR, Barr FG et al, ", Rhabdomyosarcoma and undifferentiated sarcoma in the first two decades of life: a selective review of intergroup rhabdomyosarcoma study group experience and rationale for Intergroup Rhabdomyosarcoma Study V,' J Pediatr Hematol Oncol, 2001, 23 (4): 215-20.

[21] Breneman JC, Lyden E, Pappo AS et al, ' Prognostic factors and clinical outcomes in children and adolescents with metastatic rhabdomyosarcoma -a report from the Intergroup Rhabdomyosarcoma Study IV,'” J Clin Oncol, 2003, 21 (1): 78-84.

[22] Réguerre Y, Martelli H, Rey A et al, " Local therapy is critical in localised pelvic rhabdomyosarcoma: experience of the International Society of Pediatric Oncology Malignant Mesenchymal Tumor (SIOP-MMT) committee," Eur J Cancer, 2012, 48 (13): 2020-7.

[23] Dantonello TM, Lochbühler H, Schuck A et al, ' Challenges in the local treatment of large abdominal embryonal rhabdomyosarcoma," Ann Surg Oncol, 2014, 21 (11): 3579-86.

[24] Saylors RL, Stine KC, Sullivan J et al, ' Cyclophosphamide plus topotecan in children with recurrent or refractory solid tumors: a Pediatric Oncology Group phase II study, " J Clin 
Oncol, 2001, 19 (15): 3463-9.

[25] Hull MA, Molina G, Niemierko A et al, " Improved local control with an aggressive strategy of preoperative (with or without intraoperative) radiation therapy combined with radical surgical resection for retroperitoneal sarcoma," J Surg Oncol, 2017, 115:746.

[26] Mak KS, Phillips JG, Barysauskas CM et al, " Acute gastrointestinal toxicity and bowel bag dose-volume parameters for preoperative radiation therapy for retroperitoneal sarcoma,' Pract Radiat Oncol, 2016, 6:360.

[27] Gieschen HL, Spiro IJ, Suit HD et al, “' Long-term results of intraoperative electron beam radiotherapy for primary and recurrent retroperitoneal soft tissue sarcoma,' Int J Radiat Oncol Biol Phys, 2001, 50:127.

[28] Hull MA, Molina G, Niemierko A et al, ' Improved local control with an aggressive strategy of preoperative (with or without intraoperative) radiation therapy combined with radical surgical resection for retroperitoneal sarcoma, , J Surg Oncol, 2017, 115:746.

[29] Stojadinovic A, Leung DH, Hoos A et al, " Analysis of the prognostic significance of microscopic margins in 2,084 localized primary adult soft tissue sarcomas,' Ann Surg, 2002, 235:424.

[30] Zagars GK, Ballo MT, Pisters PW et al, "' Surgical margins and reresection in the management of patients with soft tissue sarcoma using conservative surgery and radiation therapy," Cancer, 2003, 97:2544.

[31] Lehnert T, Cardona S, Hinz U et al, " Primary and locally recurrent retroperitoneal soft-tissue sarcoma: local control and survival,', Eur J Surg Oncol, 2009, 35:986.

[32] Pierie JP, Betensky RA, Choudry U et al, " Outcomes in a series of 103 retroperitoneal sarcomas,' Eur J Surg Oncol, 2006, 32:1235. 